\title{
EXCITATION OF STRUCTURES NEAR RAILWAY TRACKS-ANALYSIS OF THE WAVE PROPAGATION PATH
}

\author{
Paulius Bucinskas ${ }^{1}$ and Lars V. Andersen ${ }^{1}$ \\ ${ }^{1}$ Department of Civil Engineering, Aalborg University \\ Thomas Manns Vej 23, Aalborg 9220, Denmark \\ \{pbu,la\}@civil.aau.dk
}

Keywords: Soil vibration, wave propagation path, structure-soil interaction, railway vibrations.

\begin{abstract}
High-speed rails are an attractive alternative to other forms of intercity transportation. It is a fast, cost-efficient and environmentally friendly solution, which is being developed in various countries across the world. However, in order to be successful, high-speed rails need to transport the passengers as close as possible to the city centres. Therefore, railway tracks have to go through densely populated urban areas, which causes a number of issues. One of the biggest complaints from the inhabitants living near such infrastructures is the high vibration and noise levels caused by the passing trains.
\end{abstract}

Unfortunately, the prediction of vibrations in nearby structures is difficult, as wave propagation from the vibration source to the structure is a complex phenomenon. The behaviour of the structure is highly dependent on the path along which the vibrations travel between their source and the building itself. Especially in the densely built urban environment, the wave propagation path can have different features, such as underground infrastructure, roads, pavements or even other nearby buildings. Such features might have a significant effect on the final excitation of the structure in question.

This work aims to analyse how different features in the wave propagation path affect the excitation of a structure. A numerical model is constructed to account for the track structure and the underlying soil. The model utilizes a finite-element model for the structures together with a semi-analytical model of the underlying soil. Different features in the wave-propagation path are introduced, and their effects are compared regarding the behaviour of the structure and the free-field. 


\section{INTRODUCTION}

Continued development of railway infrastructure causes some issues related to vibrations in nearby structures. To make the railways an effective form of passenger transportation the railway stations need to be as close as possible to urban centres. This means that railway tracks need to go through densely built urban environments, causing unwanted vibrations in nearby structures. However, in situ testing of these cases is an expensive and time-consuming task which is sometimes even impossible to carry out. Therefore, computational prediction tools are needed to evaluate the effect on nearby structures. It is not an easy job as wave propagation through soil is a complex phenomenon that depends on a large number of unknowns. The ground between the source of vibrations and receiver point might often contain various obstructions. These obstructions might be on the ground surface or embedded inside the soil body, for example roads, footpaths, demolished building foundations, or sewer lines. This is especially true in a tightly built urban environment.

One of the most commonly used approaches to model the soil body is the finite-element (FE) method. Using this method it is possible to model both the structures interacting with the ground and the ground itself. However, to avoid reflections from the boundaries, absorbing boundary conditions must be used, as in the work by Connolly et al. [1], or the FE model must be coupled to a boundary-element formulation, as in the work by Andersen and Nielsen [2], Nielsen et al. [3] or Andersen [4]. Hence, a semi-analytical soil model can be beneficial to use, especially if the modelled geometry is not very complicated. The semi-analytical approach requires less computational resources and is faster to compute, and infinite boundaries are already included in the formulation. The method is based on analytical evaluation of the Green's function in frequency-wavenumber domain. The original layer-transfer matrix, used in the model, was developed by Thomson [5] and Haskell [6]. Further, the semi-analytical model is commonly used for problems involving wave propagation through soil. For example, Sheng et al. [7] used this method to analyse the vibrations from a simplified railway track placed on the soil surface.

This work also uses the semi-analytical model to model the ground, which is later coupled to structures modelled using an FE approach. The aim is to analyse the effects of various obstructions in the wave propagation path. The effects of these obstructions in the wave path might be an important factor to consider when analysing the ground-borne vibrations, and often they are completely dismissed in this type of analysis. For this, a computational model was constructed that considers a railway track coupled to the underlying ground as described in Section 2. Further, the analysed cases and parameters used are introduced in Section 3. Finally, the obtained results are presented in Section 4, and Section 5 contains the main conclusions.

\section{COMPUTATIONAL MODEL}

Problems regarding wave propagation through soil can be split into three main components: the vibration source, the propagation path and the receiver. Each of these elements has an effect on the overall system behaviour. In this paper, the vibration source is a railway track, which is introduced in Subsection 2.2. Further, the vibrations propagate through a layered soil for which a semi-analytical model is used as explained in Subsection 2.1. To reduce the number of variables, the receiver is only modelled as a rigid footing on the soil surface.

\subsection{Soil model and coupling to finite elements}

A semi-analytical approach is used to model the ground. In this case it is more advantageous compared to FE-based approaches due to lower computation times, which allows testing a wider variety of cases. The method is based on an analytical expression for the Green's function in horizontal wavenumber-frequency domain: 


$$
\mathbf{U}_{2}\left(k_{x}, k_{y}, z_{2}, \omega\right)=\mathbf{G}_{12}\left(k_{x}, k_{y}, z_{1}, z_{2}, \omega\right) \mathbf{P}_{1}\left(k_{\mathrm{x}}, k_{\mathrm{y}}, z_{1}, \omega\right) .
$$

Equation (1) provides a relation between a load $\mathbf{P}_{1}$ applied at point 1 and the corresponding displacements $\mathbf{U}_{2}$ at point 2. The Green's function $\mathbf{G}_{12}$ is dependent on the horizontal wavenumbers $k_{x}, k_{y}$ the vertical coordinates $z_{1}, z_{2}$ of both points, and the circular frequency $\omega$.

After establishing the solution, a numerical inverse Fourier transform is performed to obtain a solution in space-frequency domain. The method assumes that the soil is linear viscoelastic, composed of layers with perfectly horizontal interfaces and with a horizontal ground surface.

To couple the semi-analytical model to the FE formulation of other parts, soil-structureinteraction (SSI) nodes are used. By establishing Green's function relations between these nodes, a flexibility matrix for the soil is obtained. Inverting the flexibility matrix provides the dynamic stiffness matrix for the soil, $\mathbf{D}_{\text {soil }}$, which can then be added to the dynamic stiffness matrix of the FE-based parts, $\mathbf{D}_{\mathrm{FE}}$, to obtain an expression for the whole system:

$$
\mathbf{D}_{\text {full }}=\mathbf{D}_{\mathrm{FE}}+\mathbf{D}_{\text {soil }}, \quad \mathbf{D}_{\mathrm{FE}}=\mathbf{K}_{\mathrm{FE}}+\mathrm{i} \omega \mathbf{C}_{\mathrm{FE}}-\omega^{2} \mathbf{M}_{\mathrm{FE}},
$$

where $\mathbf{K}_{\mathrm{FE}}$ is the stiffness matrix, $\mathbf{C}_{\mathrm{FE}}$ is the damping matrix, $\mathbf{M}_{\mathrm{FE}}$ is the mass matrix, and $\mathrm{i}$ is the imaginary unit: $\mathrm{i}=\sqrt{-1}$. The calculations are then performed for the fully coupled system in frequency domain. A more detailed description of the soil model is available in the work by Andersen and Clausen [8], and coupling of multiple structures via the soil was discussed by Andersen [9] and Bucinskas et al. [10].

\subsection{Railway track and embankment model}

The structure of the railway track has a great impact on how the vibrations are transferred to the soil body. Further, the presence of the track introduces secondary effects to the system such as waves travelling through the embankment and back coupling to the surrounding structures. Therefore, proper modelling of the track structure is important.

To reduce the computational requirements of the model and decrease the computation time, shell elements were used to model the railway track and embankment. A cross-section of an embankment can be seen in Figure 1. A single plate was constructed to model the whole embankment. Mindlin-Reissner shell finite elements were used with four nodes and linear Lagrangian interpolation of the displacements and bending rotations. Two types of elements were used: an element type with three layers to model the three-layered embankment, and an element type with four layers to model the sleepers together with the embankment. Using these element types, the embankment plate is assembled to model the sleepers and all layers of the embankment.

Two rails are connected separately to the embankment. The rails are modelled as EulerBernoulli beam elements with two nodes and cubic displacement interpolation. The rails and sleepers are connected through rail pads, which are modelled as a spring and damper system. Thus, the rails are discretely supported only at the positions where the sleepers are located.

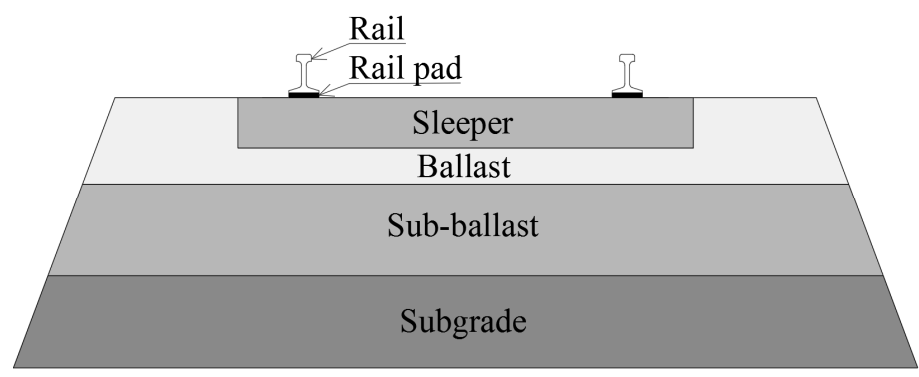

Figure 1: Railway track and embankment cross-section. 


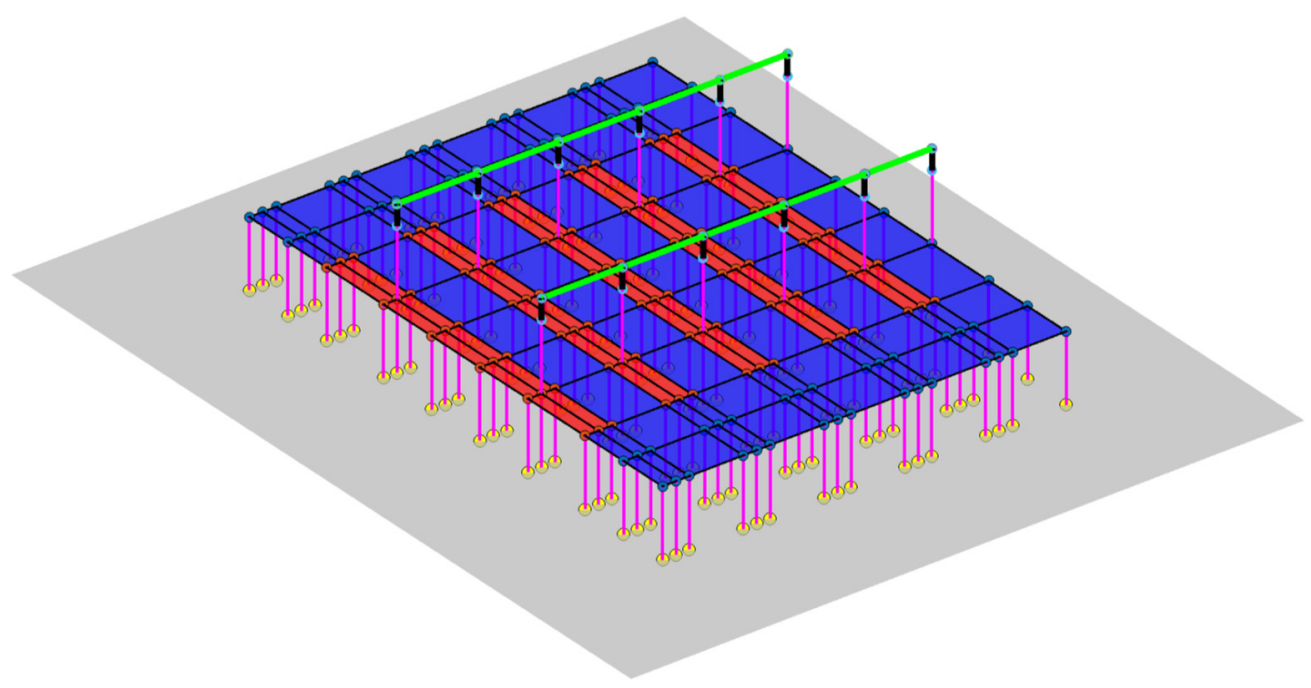

Figure 2: Parts of the track structure. In blue: three layered embankment shell elements; in red: four layered (including sleepers) embankment shell elements; in green: rail beam elements; in grey: soil surface; in yellow: SSI nodes; in magenta: rigid links between soil-embankment and embankment-rail.

The railway embankment is a three-dimensional structure with a considerable thickness. Therefore, modelling the whole embankment as a single flat plate might not be sufficient. To obtain a more realistic model, the plate was placed at the mid-plane of the embankment and then connected to the soil through rigid links. The rigid link length is equal half of the thickness of the embankment. This way the lateral displacements and rotations of the embankment nodes were coupled to lateral displacements of the SSI nodes. The same approach was then used to connect the embankment and the rail pads. The rigid links were introduced into the FE matrices using Lagrange multipliers. This approach was not difficult to implement, as the FE matrix structure stayed unchanged. An example of the FE model of the track can be seen in Figure 2.

Introducing rigid links to the system better represents the real structure, but this kind of formulation also has some disadvantages. The plate representing the embankment does not share nodes with the soil, and thus the number of degrees of freedom in the system increases significantly. Further, Lagrange multipliers also introduce additional constraints to the system, which leads to further increase of FE matrix sizes.

\section{ANALYSED CASE}

A rail track of $30.0 \mathrm{~m}$ length was modelled as described in the previous section. The embankment has a width of $4.0 \mathrm{~m}$ and is composed of three layers: a $0.5 \mathrm{~m}$ thick subgrade layer, a $0.2 \mathrm{~m}$ thick sub-ballast layer, and a $0.3 \mathrm{~m}$ thick ballast layer. The material properties for these and other materials used are given in Table 1. The sleepers are embedded in the ballast layer, and they are placed at $0.6 \mathrm{~m}$ intervals along the track. The sleepers are constructed from concrete with dimensions $0.2 \times 0.2 \times 2.5 \mathrm{~m}^{3}$. The rails are made from steel and modelled with a rectangular $0.15 \times 0.08 \mathrm{~m}$ cross-section, and the gauge is $1.4 \mathrm{~m}$. The rails are connected to the sleepers via rail pads with stiffness $1.2 \cdot 10^{8} \mathrm{~N} / \mathrm{m}$ and damping $1.24 \cdot 10^{5} \mathrm{Ns} / \mathrm{m}$.

Vibrations are measured on a rigid surface footing placed $20.0 \mathrm{~m}$ from the track structure. The rigid footing has a square shape with a side length of $3.0 \mathrm{~m}$. The footing is assumed to be $0.8 \mathrm{~m}$ thick and made from concrete. Therefore, the mass density of concrete is used to calculate the mass and mass moments of inertia, which are then added to the corresponding degrees of freedom of the rigid footing. 


\begin{tabular}{lllll}
\hline Material & Young's modulus $(\mathrm{MPa})$ & Poisson's ration $(-)$ & Mass density $\left(\mathrm{kg} / \mathrm{m}^{3}\right)$ & Damping ratio $(-)$ \\
\hline Concrete & 30000 & 0.15 & 2400 & 0.040 \\
Steel & 210000 & 0.25 & 7900 & 0.040 \\
Ballast & 100 & 0.35 & 1800 & 0.040 \\
Sub-ballast & 300 & 0.35 & 2200 & 0.040 \\
Subgrade & 120 & 0.35 & 2100 & 0.040 \\
Clay & 80 & 0.48 & 2000 & 0.045 \\
Sand & 250 & 0.30 & 2000 & 0.050 \\
\hline
\end{tabular}

Table 1: Materials and their properties used in the analysis

The whole rail track structure and the receiver footing are placed on a $4.0 \mathrm{~m}$ thick layer of clay which lies on top of a half-space of sand. This stratification was chosen because the relatively soft layer of clay over the stiffer material (sand) "traps" the propagating vibrations in the upper soil layer, leading to higher excitation at the receiver position.

The system is excited by placing a vertical unit load on one of the rails. It is placed in three different positions along the rail: Position 1 -at the centre of the track in the longitudinal direction; Position 2-6.0 m along the track from the centre; Position 3-12.0 m from the centre. In all of the positions, the load is acting on a node that is also connected to a sleeper through the rail pad. Using different positions allows analysis of the system response for cases in which the obstructions in the soil are not in the direct path of the propagating waves.

Four different cases were investigated:

- Free-field conditions. Nothing is placed between the rail track and the receiver footing. This case is used for comparison with other cases.

- A rigid box is embedded between the track and the footing, $10.0 \mathrm{~m}$ from the track. The box dimensions are $3.0 \times 3.0 \times 3.0 \mathrm{~m}^{3}$. The top of the box is embedded $1.0 \mathrm{~m}$ from the ground surface. It is assumed that the box is filled with soil and that the density of the walls is close to the density of the surrounding soil. Therefore, the additional mass is not considered.

- A single-lane road is placed on the ground surface between the source and the receiver, $10.0 \mathrm{~m}$ from the track. The road is placed alongside the track and has the same length $(30.0$ $\mathrm{m})$ as the rail track. It is constructed from concrete. The dimensions are: width $4.0 \mathrm{~m}$ and thickness $0.3 \mathrm{~m}$. The road structure is modelled using Mindlin-Reissner shell finite elements coupled to soil through SSI nodes.

- A concrete pipe is placed alongside the rail track, $10.0 \mathrm{~m}$ from the track and embedded $1.5 \mathrm{~m}$ into the soil. As in the previous case, the pipe runs alongside the track and has the same length. The pipe is modelled as a cylindrical tube with an outer diameter of $1.0 \mathrm{~m}$ and an inner diameter of $0.75 \mathrm{~m}$. It is modelled as a finite Euler-Bernoulli beam coupled to the soil through SSI nodes.

- Same as previous case, just this time the pipe is oriented at $45^{\circ}$ angle to the rail track. The pipe centre is $10 \mathrm{~m}$ from the track.

- Once again the same embedded pipe is used. In this case, the pipe is at $90^{\circ}$ angle to the track, that is the pipe goes under the rail track and extends towards the receiver footing.

\section{THE EFFECT OF OBSTRUCTIONS IN THE WAVE PROPAGATION PATH}

The wave propagation path was analysed for the previously described six cases. The analysis was performed from $1 \mathrm{~Hz}$ up to $50 \mathrm{~Hz}$, with $1 \mathrm{~Hz}$ intervals. The results are presented in Figures 3 and 4. 


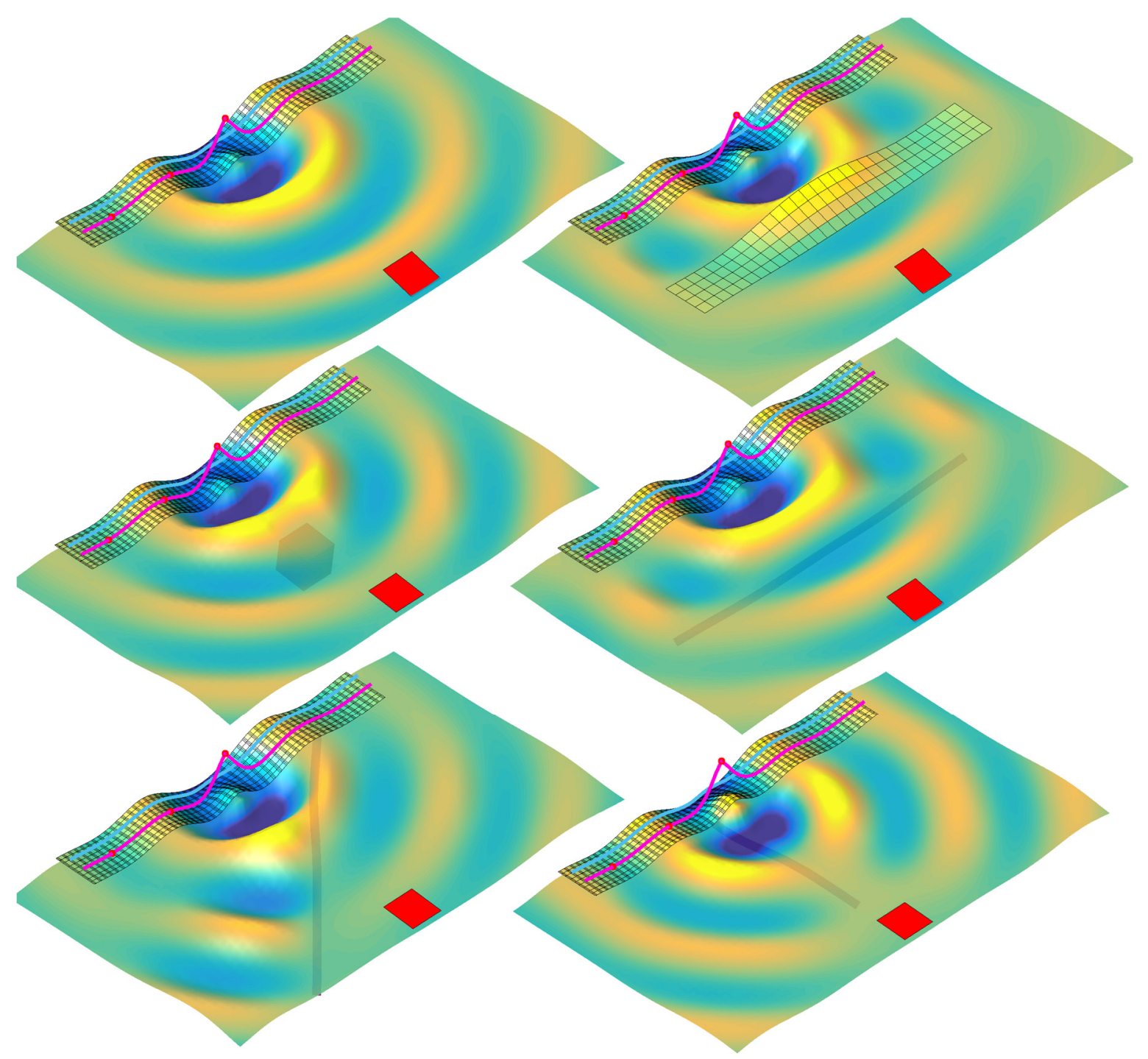

Figure 3: Steady state soil surface response for a load applied on the rail at the centre of the track, excited at $25 \mathrm{~Hz}$. Blue/yellow shades indicate positive/negative displacement in vertical direction. The red dots on the rail show the three positions in which the load is applied within the analysis. In this case, the load is only applied in the first point. Top left: free-field; top right: road placed between the track and receiver; middle left: embedded rigid box; middle right: embedded pipe along the track; bottom left: embedded pipe at $45^{\circ}$ angle to the track; bottom right: embedded pipe at orthogonal to the track.

Figure 3 shows the response of the soil surface excited at $15 \mathrm{~Hz}$. All cases are shown for the load placed on the rail at Position 1. It is evident that the displacements of the soil are effected significantly by obstructions in the wave propagation path. The road surface forms a waveimpeding barrier for the propagating surface waves, thus decreasing the displacement field over a large area. However, the effect at the receiver footing is rather small. Further, the embedded rigid box has a larger effect for the receiver footing, however it is localized to a small shadow area behind the box. The overall effect to the displacement is much smaller when compared to the road case. Further, all three cases of embedded pipes cause very different behaviour of the system. For the pipe laid along the track, the effects are very similar to the road case - the displacement field is reduced in a large area, but the effects at the receiver footing are small. It can be seen that obstructions have less impact when the obstructions are laid orthogonally to the propagating waves. This is illustrated in the next case-embedded pipe at $45^{\circ}$ angle. In this 
case there is a significant reduction of displacements at the receiver footing, while the displacements are increased directly in front of the embedded pipe. Finally, the last case, the embedded pipe orthogonal to the track, provides the most significant reduction of displacements for the receiver footing. However, similar to the embedded box, the effects are localized to a smaller area and the surrounding displacement field is largely unaffected.

Further, Figure 4 shows the absolute velocities at the receiver position for all cases. Overall it can be seen that introducing obstructions in the wave propagation field have the highest effect in frequency ranges between $7-40 \mathrm{~Hz}$, while frequencies up to $7 \mathrm{~Hz}$ are almost unaffected. In the $7-30 \mathrm{~Hz}$ range, the obstructions tend to reduce the velocities at the receiver position when compared to the free-field case. However, in the $30-40 \mathrm{~Hz}$ range the effects are not as evident. In this range, some cases - especially the road — tend to increase the velocities significantly.

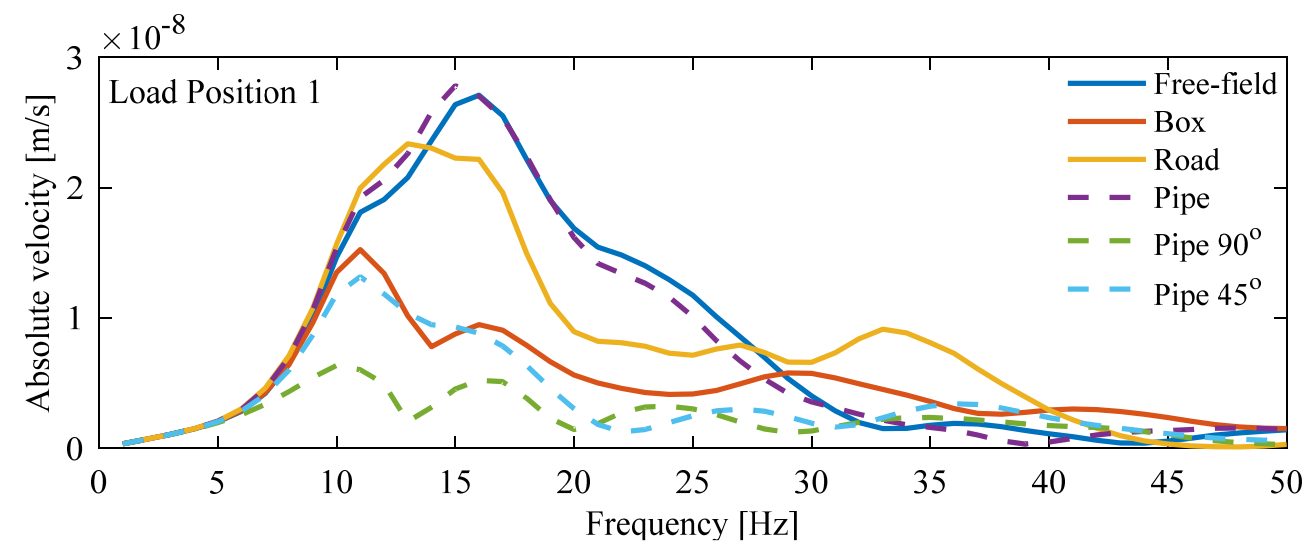

(a)

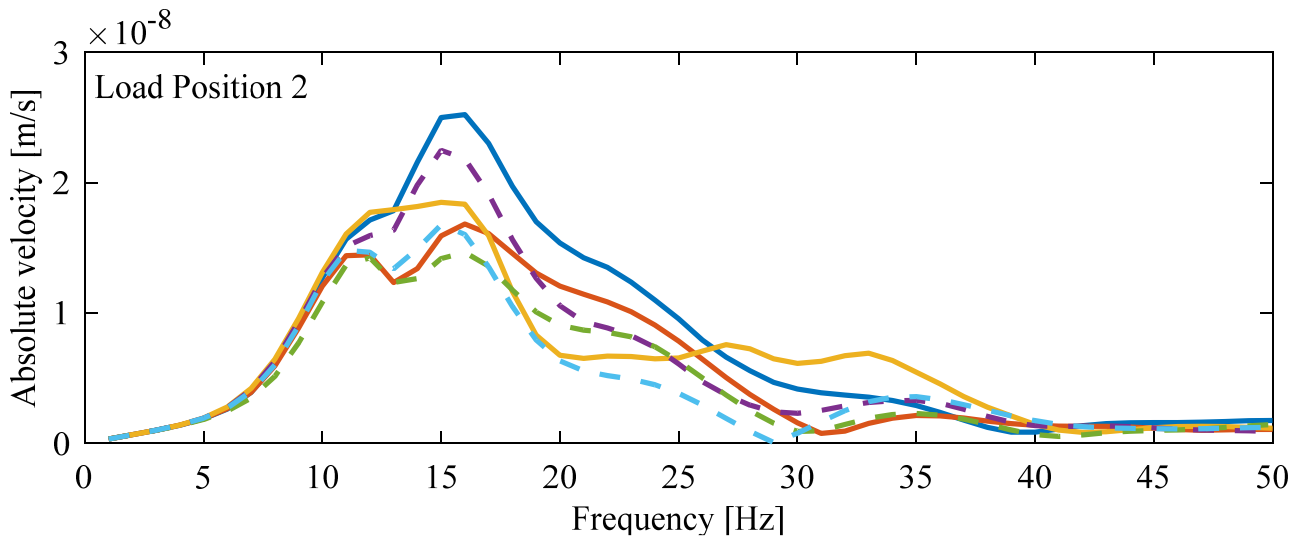

(b)

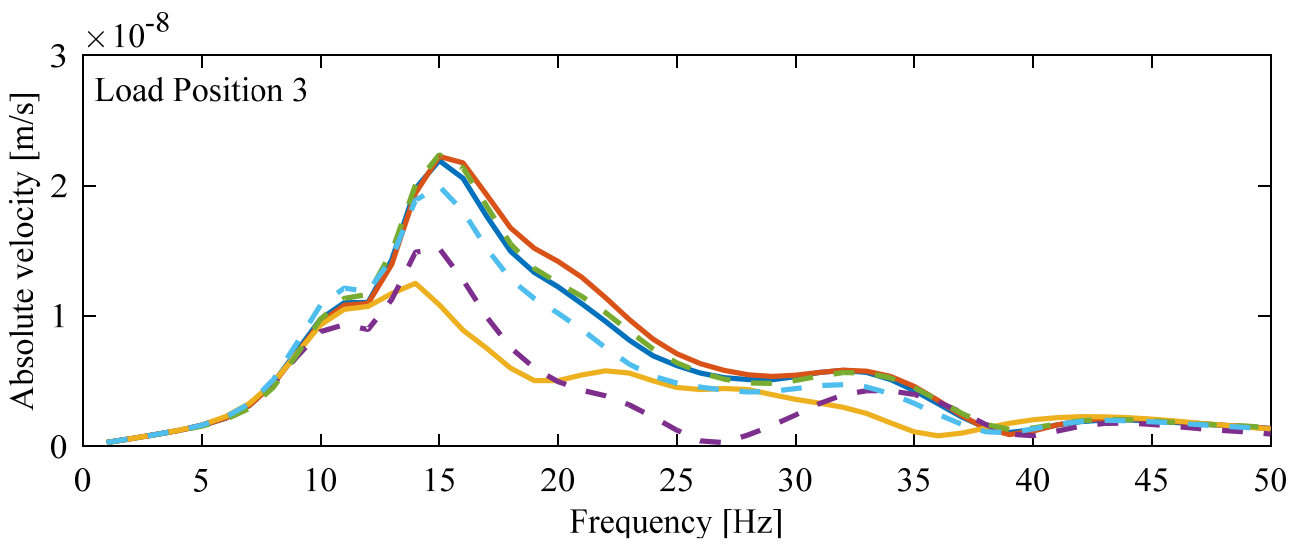

(c)

Figure 4: Response at the receiver for obstructions in the wave propagation path: a) load Position 1, applied on a rail at the centre of track; b) load Position 2, applied on a rail $6.0 \mathrm{~m}$ from the centre of the track;

c) load Position 3, applied on a rail $12.0 \mathrm{~m}$ from the centre of the track. 
Further, Figure 4 shows that pipe running along the rail track has a very small effect when the load is placed at Position 1. However, this effect increases when the load is placed further from the track centre. A completely opposite behaviour is observed with the pipe placed orthogonally to the track - the effects become smaller when the centre the load is placed further away from the track. This shows that the angle at which the waves reach the obstruction is a very important factor in the analysed system. The road case has a similar effect as the pipe placed along the track, but the overall effects across all loading positions are higher. This is due to larger stiffness and mass introduced to the system by the road, when compared to an embedded pipe. However, in the $30-40 \mathrm{~Hz}$ frequency range, the changes in absolute velocity relative to the reference case become positive, that is the velocities at the receiver increase when compared to the free-field case. Finally, the rigid box has a more localized effect. For loading in Positions 1 and 2, the effects are positive and rather large, but as soon as the box is not in the direct wave path between source and receiver (loading at Position 3 ) these effects disappear almost completely.

\section{CONCLUSIONS}

A presentation has been given of ground vibration due to harmonic excitation. Analysis was performed to investigate the effects of obstacles placed inside the soil body or on the ground surface between the source and the receiver. The load was applied on a railway track and the response was checked on a surface footing. Five different cases were tested: an embedded rigid box (simulating an infilled tank or former cellar), a segment of a single-lane road (or a large footpath) running parallel to the rail track on the soil surface, and three different orientations of an embedded pipe in the wave propagation path. The cases were compared to the free-field response with no obstacles in or on the ground. A computational model was created using an FE formulation to model the rail track structure and the obstructions, while the soil was modelled using a semi-analytical approach.

The results show that obstruction in the wave propagation field does cause significant changes in the overall system behaviour. In most cases, the obstacles have a mitigation effect, thus reducing the absolute velocities, especially within the $7-30 \mathrm{~Hz}$ frequency range. However, in some cases, the response increases within the 30-40 Hz frequency range. The effects of the structures considered in this paper are mostly seen at higher frequencies. This can be explained by the relatively small size of the considered structures, where at lower frequencies the wavelength in the soil body is much longer than the dimensions of the structures. Therefore, the effects at low frequencies are very small.

Future work could investigate these effects for a wider frequency range together with different soil-stratification cases. Further, analysis could be performed for different obstructions to the propagating waves that are commonly present, such as: tree roots, large boulders, underground cables, etc. Also more in-depth analysis of different orientations of the structures considered might reveal different effects caused to the system.

\section{ACKNOWLEDGMENTS}

The research was carried out in the framework of the project "Urban Tranquility" under the Interreg V programme. The authors of this work gratefully acknowledge the European Regional Development Fund for the financial support. 


\section{REFERENCES}

[1] D. Connolly, A. Giannopoulos, and M. C. Forde. Numerical modelling of ground borne vibrations from high speed rail lines on embankments. Soil Dynamics and Earthquake Engineering 46: 13-19, 2013.

[2] L. Andersen and S. R. K. Nielsen, Reduction of ground vibration by means of barriers or soil improvement along a railway track, Soil Dyn. Earthq. Eng., 25(7-10), 701-716, 2005.

[3] J.C.O. Nielsen, G. Lombaert, and S. François. A hybrid model for prediction of groundborne vibration due to discrete wheel/rail irregularities. Journal of Sound and Vibration 345: 103-120, 2015.

[4] L.V. Andersen, Influence of dynamic soil-structure interaction on building response to ground vibration, in Numerical Methods in Geotechnical Engineering-Proceedings of the 8th European Conference on Numerical Methods in Geotechnical Engineering, NUMGE 2014, 2014, vol. 2.

[5] W.T. Thomson, Transmission of elastic waves through a stratified solid medium, Journal of applied Physics 21.2: 89-93, 1950.

[6] N.A. Haskell, The dispersion of surface waves on multilayered media, Bulletin of the seismological Society of America 43.1: 17-34. 1953.

[7] X. Sheng, C. J. C. Jones, and M. Petyt, Ground vibration generated by a harmonic load acting on a railway track, Journal of sound and vibration 225.1: 3-28, 1999.

[8] L. Andersen and J. Clausen, Impedance of surface footings on layered ground, Computers \& structures 86.1: 72-87, 2008.

[9] L. V. Andersen, "Dynamic soil-structure interaction of monopod and polypod foundations," in Insights and Innovations in Structural Engineering, Mechanics and Computation, Proceedings of the Sixth International Conference on Structural Engineering, Mechanics and Computation, 5-7 September 2016, Cape Town, South Africa, 2016, pp. 2036-2041.

[10] P. Bucinskas, L. V. Andersen, and K. Persson, "Numerical modelling of ground vibration caused by elevated high-speed railway lines considering structure-soil-structure interaction," in Proceedings of the INTER-NOISE 2016 - 45th International Congress and Exposition on Noise Control Engineering: Towards a Quieter Future, 2016. 